\title{
Radiation-induced lung metastasis development is MT1-MMP-dependent in a triple-negative breast cancer mouse model
}

\author{
Gina Bouchard ${ }^{1}$, Hélène Therriault ${ }^{1}$, Sameh Geha ${ }^{2}$, Rachel Bujold ${ }^{1,3}$, Caroline Saucier ${ }^{4}$ and Benoit Paquette ${ }^{*, 1}$ \\ ${ }^{1}$ Centre for Research in Radiotherapy, Department of Nuclear Medicine and Radiobiology, Faculty of Medicine and Health \\ Sciences, Sherbrooke, Québec, Canada; ${ }^{2}$ Department of Pathology, Centre Hospitalier Universitaire de Sherbrooke, Sherbrooke, \\ Québec, Canada; ${ }^{3}$ Service of Radiation Oncology, Centre Hospitalier Universitaire de Sherbrooke, Sherbrooke, Québec, Canada \\ and ${ }^{4}$ Department of Anatomy and Cellular Biology, Faculty of Medicine and Health Sciences, Universite de Sherbrooke, \\ Sherbrooke, Québec, Canada
}

Background: The prognosis of triple-negative breast cancer (TNBC) is still difficult to establish. Some TNBC benefit from radiotherapy (RT) and are cured, while in other patients metastases appear during the first 3 years after treatment. In this study, an animal model of TNBC was used to determine whether the expression of the cell membrane protease MT1-MMP in cancer cells was associated with radiation-stimulated development of lung metastases.

Methods: Using invasion chambers, irradiated fibroblasts were used as chemoattractants to assess the invasiveness of TNBC D2A1 cell lines showing downregulated expression of MT1-MMP, which were compared with D2A1-wt (wild-type) and D2A1 shMT1-mock (empty vector) cell lines. In a mouse model, a mammary gland was irradiated followed by the implantation of the downregulated MT1-MMP D2A1, D2A1-wt or D2A1 shMT1-mock cell lines. Migration of D2A1 cells in the mammary gland, number of circulating tumour cells and development of lung metastases were assessed.

Results: The reduction of MT1-MMP expression decreased the invasiveness of D2A1 cells and blocked the radiation enhancement of cancer cell invasion. In BALB/c mice, irradiation of the mammary gland has stimulated the invasion of cancer cells, which was associated with a higher number of circulating tumour cells and of lung metastases. These adverse effects of radiation were prevented by downregulating the MT1-MMP.

Conclusions: This study shows that the MT1-MMP is necessary for the radiation enhancement of lung metastasis development, and that its expression level and/or localisation could be evaluated as a biomarker for predicting the early recurrence observed in some TNBC patients.

Triple-negative breast cancer (TNBC; oestrogen, progesterone and human epidermal growth factor receptors negative) is not a homogeneous group in terms of their recurrence-free survival. In patients showing a pathological complete remission (pCR) after treatments, a rapid recurrence is observed for some of them within the first 40 months, whereas $70-80 \%$ are still disease-free 10 years later (Loibl et al, 2015; Villarreal-Garza et al, 2016). These findings support the idea that it would be important to count on a biomarker to identify TNBC at high risk of early recurrence to propose a more efficient treatment.

No clinical trials have been designed to determine whether radiation therapy could increase the risk of early recurrence in certain subgroups of patients. However, studies performed in animal models support the idea that radiation stimulates the invasion of cancer cells and the development of metastases; which has been shown as follows: (1) after irradiation of the

*Correspondence: Professor B Paquette; E-mail: Benoit.Paquette@USherbrooke.ca

Revised 5 December 2016; accepted 15 December 2016; published online 19 January 2017

(c) 2017 Cancer Research UK. All rights reserved 0007-0920/17 
hepatocellular tumour MHCC97L or the Lewis lung tumour (Jia et al, 2011; Chou et al, 2012), (2) by irradiating healthy tissue before implantation of non-irradiated MC7-L1 or D2A1 breast carcinoma cells or F98 glioma cells (Lemay et al, 2011; Bouchard et al, 2013; Desmarais et al, 2015) and (3) when 9L or F98 glioma cells were irradiated before their implantation in animals (Wild-bode et al, 2001; Desmarais et al, 2012). Interestingly, this adverse effect of radiation is not observed in all the cancer cell lines, but only in some subgroups, which are still poorly identified (Wang et al, 2000; Wild-bode et al, 2001; Qian et al, 2002; Rofstad et al, 2004; Speake et al, 2005; Paquette et al, 2011).

Considering that a subgroup of TNBC patients could potentially be resistant to radiotherapy and a recurrence rapidly developed (Podo et al, 2010; Langlands et al, 2013), it is relevant to determine whether early recurrence in these TNBC patients could be related to the ability of radiation to stimulate metastasis development.

The aim of the present study is to determine whether the membrane-type matrix metalloproteinase-1 (MT1-MMP) is associated with a higher propensity to develop lung metastases after irradiation in an animal model of TNBC. MT1-MMP was selected because of its central role in cancer cell invasion by activating the metalloproteinases-2 and -9 (MMP-2 and MMP-9) that cleave extracellular matrix (ECM) proteins and thus opening a passage to cancer cells (Egeblad and Werb, 2002; Hotary et al, 2002). ECM proteins can also be directly cleaved by MT1-MMP located at the leading edge of the cancer cells (Itoh, 2006). In TNBC patients, high levels of MT1-MMP were correlated with blood vessel invasion of cancer cells (Perentes et al, 2011) and were also associated with shorter overall survival (Li et al, 2015). In a preclinical mouse model, MT1-MMP blockade with a specific antibody decreased the growth of 4T1 TNBC tumours and synergistically enhanced tumour response to radiation therapy (Ager et al, 2015).

When designing the animal assays, it was decided to evaluate the role of MT1-MMP in cancer cells implanted in mammary glands already irradiated. This experimental setup excludes the possibility that new mutations induced by radiation would have modified the invasiveness of TNBC cells. This model also allows to better understand the association between radiation-induced inflammation in the mammary gland, the level of MT1-MMP in TNBC cells and the development of lung metastases.

\section{MATERIALS AND METHODS}

Cell culture. The mouse breast carcinoma D2A1 cells, kindly provided by Dr Ann F. Chambers (University of Western Ontario, London, ON, Canada), were derived from a spontaneous mammary tumour in a $\mathrm{BALB} / \mathrm{c}$ mouse and the mouse $3 \mathrm{~T} 3$ fibroblasts were obtained from the American Type Culture Collection (ATCC, Manassas, VA, USA). TNBC status of the D2A1 cells was confirmed by a certified pathologist, SG, from the Centre Hospitalier Universitaire de Sherbrooke, using standard immunohistochemistry protocols suitable for the detection of mouse oestrogen receptor (ER), progesterone receptor (PR) and HER2. The cells were maintained in a $5 \% \mathrm{CO}_{2}$ humidified incubator at $37^{\circ} \mathrm{C}$ in Dulbecco's modified Eagle's medium (DMEM; Sigma-Aldrich, Oakville, ON, Canada) supplemented with $10 \%$ fetal bovine serum (FBS; Wisent, St Bruno, QC, Canada), $2 \mathrm{~mm}$ glutamine, $1 \mathrm{~mm}$ sodium pyruvate, 100 units per $\mathrm{ml}$ penicillin and $100 \mathrm{~mm}$ streptomycin.

Downregulation of MT1-MMP expression by short hairpin RNA. For the production of lentiviral particles, the human cell line $293 \mathrm{~T}$ was co-transfected using lipofectamine with $6 \mu \mathrm{g}$ of the plp1, plp2 and plp/VSV-G plasmids (Invitrogen, Burlington, ON, Canada) and $6 \mu \mathrm{g}$ of pLKO.1-puro vector containing a short hairpin RNA (shRNA) sequence targeting murine MT1-MMP transcript (Sigma-Aldrich, Oakville, ON, Canada: TRCN000 0031264, TRCN0000031265, TRCN0000031266, TRCN00000 31267). The pLKO empty vector was used as a negative control (mock cells). After $48 \mathrm{~h}$, the lentivirus-containing supernatant (cell media) was collected and filtered with a $0.45 \mu \mathrm{m}$ membrane and then kept at $-80^{\circ} \mathrm{C}$ for further use. The D2A1 cells expressing the fluorescent proteins FUCCI were prepared as previously described (Bouchard et al, 2013). The D2A1 FUCCI cells were infected with $700 \mu \mathrm{l}$ of the virus-enriched supernatant containing $4 \mu \mathrm{g} \mathrm{ml}^{-1}$ polybrene (Sigma-Aldrich, Oakville, ON, Canada). After $48 \mathrm{~h}$ incubation, DMEM containing the virus was replaced with fresh DMEM containing $1.5 \mu \mathrm{g} \mathrm{ml}^{-1}$ of puromycin as selection agent and incubated for 10 days.

Validation scheme for downregulation of MT1-MMP is described in Supplementary Methods. Briefly, quantitative Polymerase Chain Reaction (qPCR) was used to confirm MT1-MMP mRNA (Mmp14) knockdown (Supplementary Figure 1A), and the decrease in MT1MMP protein level was confirmed by western blot (Supplementary Figure $1 \mathrm{~B}$ and $\mathrm{C}$ ). Reduction of MMP-2 activation, the main extracellular substrate of MT1-MMP, was shown by zymogram analysis (Supplementary Figure $1 \mathrm{D}$ and E). Nomenclature of the derived D2A1 cell lines is as follows: D2A1-wt (wild-type), D2A1 shMT1-mock (pLKO empty vector), D2A1 shMT1-40 (40\% downregulation of the Mmp14 transcript level) and D2A1 shMT1-70 (70\% downregulation of the Mmp14 transcript level).

Invasion capacity of $\mathrm{D} 2 \mathrm{~A} 1$ cells assessed in invasion chambers. Invasion capacity of D2A1 cells expressing different levels of MT1-MMP was assessed in invasion chambers as previously described (Bouchard et al, 2016). Briefly, BALB/c 3T3 fibroblasts $\left(2.5 \times 10^{4}\right)$ were seeded with MEM supplemented with $10 \%$ FBS in 24 -well plates. After $20 \mathrm{~h}$, the cell culture medium was replaced with MEM supplemented with $0.1 \%$ bovine serum albumin (BSA) following two rinses in PBS. The cells were then irradiated using a ${ }^{60}$ Co source (Gammacell 220, Nordion, Laval, QC, Canada) at a dose of $5 \mathrm{~Gy}$. The fibroblast-conditioned media were harvested $24 \mathrm{~h}$ later, and used as chemoattractant in the lower compartment of the invasion chambers (Becton Dickinson Biosciences, Bedford, MA, USA). Sham-irradiated cells were used as control. Invasion chambers coated with Matrigel (artificial basement membrane, $8-12 \mathrm{mg} \mathrm{ml}^{-1}$ ) were rehydrated with $1 \mathrm{ml} \mathrm{DMEM} 0.1 \%$ BSA for $2 \mathrm{~h}$ at $37^{\circ} \mathrm{C}$. Nonirradiated D2A1 cells harvested with Cell Dissociation Solution (Sigma-Aldrich, Oakville, ON, Canada) were added $\left(4 \times 10^{4}\right)$ to the upper compartment of the invasion chambers. D2A1 cells that had passed across the Matrigel and the porous membrane ( $8 \mu \mathrm{m}$ pores) $24 \mathrm{~h}$ later were fixed, stained with crystal violet and counted under the microscope. The invasion capacity of the MT1-MMP wild-type and downregulated cells were compared. The results were reported as radiation-enhancement ratio and normalised using non-irradiated D2A1-wt cells as control $(n=6)$.

Mammary gland irradiation. The experimental protocols were approved by the Université de Sherbrooke Ethics Committee for Animal Care and Use in accordance with guidelines established by the Canadian Council on Animal Care (Protocol ID number 013-14). BALB/c female retired breeder mice (18-24 weeks old) were obtained from Charles River. The animals were anaesthetised with 3\% isoflurane and then immobilised with a stereotactic mouse frame adapted to dock on the Leskell Gamma Knife Perfexion (Elekta, Stockholm, Sweden). The third right mammary gland was irradiated by an energy deposition of elliptical shape as previously described (Bouchard et al, 2013). Anaesthetised mice were irradiated daily with $6 \mathrm{~Gy}$ for a total of four fractions. Based on dosimetry performed by our institutional medical physicist team, this protocol provided a biological effective dose (BED) comparable to the standard clinical regimen of $20 \times 2.25 \mathrm{~Gy}$, without having to perform daily anaesthesia over 20 days that would most likely be lethal for mice. Regarding the third left 
mammary gland (non-irradiated control) in the same animal, a residual dose of $<1 \%$ was received.

Implantation of D2A1 FUCCI expressing cells in mammary glands. D2A1 FUCCI cells ( $10^{6}$ per $100 \mu \mathrm{l}$ PBS) expressing different levels of MT1-MMP were implanted in pre-irradiated (right side) and in the opposite non-irradiated (control; left side) mammary glands $3 \mathrm{~h}$ after the last irradiation. The D2A1 cells were also implanted into the mammary glands of sham-irradiated mice, and the number of circulating tumour cells and lung metastases were compared with those of irradiated animals. Overall, eight groups of mice were assessed; SHAM + D2A1-wt $(n=22)$, IRR + D2A1-wt $(n=24)$, SHAM + D2A1 shMT1-mock $(n=9)$, IRR + D2A1 shMT1-mock $(n=11)$, SHAM + D2A1 shMT1-40 $(n=9)$, IRR + D2A1 shMT1-40 $(n=9)$, SHAM + D2A1 shMT1-70 $(n=8)$ and IRR + D2A1 shMT1-70 $(n=8)$. To exclude possible systemic effects of radiation on tumour growth, tumour volumes of shamirradiated animals (sham tumours) were compared with control tumours (left side) of irradiated animals as a validation of the mice as its own control in following experiments (Supplementary Figure 2). Tumour volumes were measured every 3 days using a caliper and calculated with the formula: $V\left(\mathrm{~mm}^{3}\right)=\pi / 6 \times a$ $(\mathrm{mm}) \times b^{2}\left(\mathrm{~mm}^{2}\right)$, where ' $a$ ' and ' $b$ ' are the largest and smallest perpendicular tumour diameters.

Quantification of pulmonary metastases by optical imaging. Fluorescence emitted by D2A1 FUCCI-expressing cells were used to quantify the number of lung metastases using an animal optical imager (QOS Imager; Quidd S.A.S., Val de Reuil, France). On day 21 , mice were killed and their lungs removed. A bright-field image of the lungs was taken and then the appropriate filters were selected for red and green fluorescence acquisition $(\mathrm{mKO} 2$, $\lambda \mathrm{ex}=472 / 30, \lambda \mathrm{em}=536 / 40 ; \mathrm{mAG}, \lambda \mathrm{ex}=531 / 40, \lambda \mathrm{em}=593 / 40$ ). These three images were merged for manual quantification of lung metastases.

Histology. Mammary tumours and lung specimens containing D2A1 FUCCI-expressing cells were dissected and frozen in optimal cutting temperature compound (OCT; Electron Microscopy Sciences, Hatfield, PA, USA) for cryosections or fixed with $4 \%$ paraformaldehyde (PFA) before paraffin embedding. After H\&E staining, primary tumour and infiltrated areas in fixed specimens sections were delimited microscopically with a Nanozoomer (Hamamatsu, Hertfordshire, UK) using Nanozoomer Digital Pathology software. Migration ratio was quantified as infiltrating area/primary tumour area, and control tumours were compared with the tumours implanted in pre-irradiated mammary glands.

Cryosections of $3 \mu \mathrm{m}$ were cut using a Leica CM3050 Microsystems cryostat $(\mathrm{GmbH}$, Wetzlar, Germany). The slides were dried for $30 \mathrm{~min}$ at $37^{\circ} \mathrm{C}$ and then stored at $-80^{\circ} \mathrm{C}$ until further use. The fluorescence emitted by the D2A1 cells was recorded using the FSX100 Bio Imaging Navigator microscope (Olympus, Center Valley, PA, USA) equipped with band pass filters (Chroma Technology Corp, Bellows Falls, VT, USA) for fluorescein isothiocyanate (FITC (green); $\lambda$ ex $=480 / 30$, $\lambda \mathrm{em}=535 / 40$ ) or tetramethylrhodamine isothiocyanate (TRITC (red); $\lambda \mathrm{ex}=560 / 40, \lambda \mathrm{em}=630 / 60)$. The whole slide was scanned (magnification $\times 42$ ) and the ratio of red and green fluorescence intensity emitted by the tumour cells was calculated. The immunohistochemistry (IHC) was performed as described in Supplementary Methods and Supplementary Figure 3.

Western blot. Protein extractions were performed from tumour homogenates in lysis buffer containing $50 \mathrm{~mm}$ Tris- $\mathrm{HCl}, \mathrm{pH} 7.5$, $150 \mathrm{~mm} \mathrm{NaCl}, 0.1 \%$ sodium dodecyl sulfate, $1 \% \mathrm{NP}-40,0.5 \%$ $\mathrm{Na}$-deoxycholate and $5 \mathrm{~mm}$ EDTA supplemented with the protein inhibitor cocktail Complete Mini, EDTA-free (Roche Diagnostics, Indianapolis, IN, USA). Cellular debris were cleared by centrifugation and supernatants were aliquoted and stored at $-80^{\circ} \mathrm{C}$ for further use. Protein quantification assay was performed with a DC Protein Assay kit (Bio-Rad, Hercules, CA, USA). The protein extracts were applied on a $12 \%$ polyacrylamide-SDS gel at $120 \mathrm{~V}$ for $3 \mathrm{~h}$ at $4{ }^{\circ} \mathrm{C}$ and transferred to a PVDF membrane (EMD Millipore, Billerica, MA, USA) using the Mini Trans-Blot Cell (Bio-Rad, Hercules, CA, USA) settled at $160 \mathrm{~V}$ for $1 \mathrm{~h}$. The membrane was blocked with $8 \%$ reconstituted skim milk powder in TBST solution (10 mM Tris- $\mathrm{HCl}$ pH 7.5 containing $150 \mathrm{~mm} \mathrm{NaCl}$ and $0.05 \%$ Tween 20). The blots were incubated with MT1-MMP ( $1: 750$, sc30074, Santa Cruz Biotechnology, Santa Cruz, CA, USA) or HIF- $1 \alpha$ antibodies $(1: 200$, NB-100-654; Novus Biologicals, Oakville, ON, Canada) in blocking solution overnight at $4{ }^{\circ} \mathrm{C}$. After washing with TBST, rabbit horseradish peroxidaseconjugated secondary antibodies (1:10 000, LS-C181152, LifeSpan BioSciences, Seattle, WA, USA) were applied and the blots developed by the Enhanced Chemiluminescence Detection System (Perkin Elmer, Waltham, MA, USA). Relative intensity of the bands were normalised to $\alpha$-tubulin internal standard.

Circulating tumour cells. Blood samples were collected from the lateral saphenous vein of the sham and irradiated mice at days 4 and 7 after the implantation in the mammary glands of the D2A1 FUCCI-labelled cells expressing different levels of MT1-MMP. Approximately 5 to $10 \mu \mathrm{l}$ of blood was collected using capillary blood collection tubes. Samples diluted 1:10 in PBS were spread in a $35 \mathrm{~mm}$ Petri dish and covered with a glass cover slip for quantification. The presence of circulating tumour cells (CTC) in each blood sample was quantified by fluorescence microscopy from five images of representative areas (magnification $\times 100$ ) that were acquired as described above with an Olympus fluorescence microscope. Fluorescence microscopy method was chosen over FACS analysis, allowing repeated quantification with very small blood volumes. The results were plotted as a radiation-enhancement ratio of CTC and calculated as the mean number of CTC/ field in the blood of irradiated animals divided by the mean number of CTC/field quantified in the blood of sham animals.

Statistical analysis. Experimental data are shown as mean \pm standard error mean (s.e.m.). Statistical analyses were performed using one- or two-way analysis of variance (ANOVA) with multiple comparisons Fisher's least significant difference (LSD) test where each comparison stands alone. A $P$ value of 0.05 was considered to be statistically significant. ${ }^{\star} P<0.05,{ }^{*} P<0.01$, ${ }^{* * *} P<0.001$ and ${ }^{* * * *} P<0.0001$.

\section{RESULTS}

MT1-MMP promotes radiation-stimulated invasion in vitro. To determine whether MT1-MMP is involved in radiation-stimulated invasion of D2A1 breast cancer cells, two stable cell lines expressing lower levels of MT1-MMP were prepared using shRNA. Downregulation of $40 \%$ and $70 \%$ were achieved as assessed by quantifying mRNA by qPCR (Supplementary Figure 1A). Protein expression was also quantified by western blot analyses (Supplementary Figure 1B and C) and reduction of the enzymatic activity of MT1-MMP was confirmed by measuring the conversion of proMMP-2 to MMP-2 by zymography (Supplementary Figure $1 \mathrm{D}$ and $\mathrm{E})$. A third cell line was generated with the empty plasmid as a negative control (mock). The cell lines used in this study were identified as D2A1-wt (wild-type), D2A1 shMT1-mock (pLKO empty vector), D2A1 shMT1-40 (40\% downregulation of MT1-MMP mRNA transcript) and D2A1 shMT1-70 (70\% downregulation of MT1-MMP mRNA transcript).

The invasiveness of these cell lines was first assessed with invasion chambers coated with Matrigel, and using the BALB/c 3T3 fibroblasts irradiated with $5 \mathrm{~Gy}$ as chemoattractant in the lower compartment of the chamber. The invasiveness of the D2A1-wt cells was increased 
by 1.6-fold compared with assays realised with non-irradiated 3T3 cells $(P<0.0001$; Figure 1A). Reduction of MT1-MMP expression decreased the basal invasion capacity of the D2A1 cells. The invasiveness of the D2A1 shMT1-40 was reduced by $32 \%$ $(P=0.0108)$ and by $63 \%$ for the D2A1 shMT1-70 cells $(P<0.0001)$. More significant, the ability of radiation to promote the invasion of D2A1 cells was completely inhibited for both MT1-MMP downregulated cell lines. These results support the important role of MT1-MMP in invasion as well as radiationstimulated invasion of D2A1 breast cancer cells in vitro.

MT1-MMP expression affects the growth of D2A1 tumours implanted in pre-irradiated mammary glands. The D2A1 cell lines were implanted in mammary glands of $\mathrm{BALB} / \mathrm{c}$ mice that were pre-irradiated or not (control mammary glands). No difference in tumour volumes was measured with the four cell lines (D2A1-wt, shMT1-mock, shMT1-40 and shMT1-70) when implanted in non-irradiated mammary glands (solid lines; Figure 1B). In contrast, 3 weeks after implantation, tumour volumes were significantly decreased for these four cells lines when implanted in pre-irradiated mammary glands (dotted lines; $P<0.0001$ ), a reduction that was even more significant for the MT1-MMP downregulated tumours (Figure 1B). Although the shMT1-40 tumours seemed to be smaller than the shMT1-70 tumours, their respective volumes were not statistically different $(P=0.1819)$. Reduction of tumour growth when implanted in pre- irradiated mammary glands was not associated with a decrease of mitotic activity as no significant difference in Ki67 positive cells (proliferation marker) was measured between all D2A1 tumours (Figure $1 \mathrm{C}$ and D).

MT1-MMP downregulation decreases tumour vascularisation. Modifications of the vascularisation can affect tumour growth and invasion of cancer cells. The impact of MT1-MMP knockdown on tumour vascularisation was then assessed by quantifying the endothelial cell marker CD31 by IHC on tumour frozen sections. A significant decrease $(36 \%, P<0.0001)$ in CD31 staining was measured only in the D2A1 shMT1-70 tumours (Figure 2). A reduction of $\mathrm{CD} 31$ staining was also observed in the D2A1 shMT1-40 tumours, but the decrease was not statistically significant. Diminution of the blood vessel area was independent of the irradiated microenvironment since no difference was observed between control tumours and those implanted in pre-irradiated mammary glands.

The effect of the downregulation of MT1-MMP on hypoxia inducible factor 1 alpha (HIF-1 $\alpha$ ) expression was also assessed by western blot on D2A1 tumours sections, as MT1-MMP is known to segregate its main inhibitor, the factor inhibiting HIF- $1 \alpha(\mathrm{FIH}-$ $1 \alpha$ ) at the Golgi membrane. HIF- $1 \alpha$ is an indicator of hypoxia, which induces the recruitment of endothelial progenitor cells, promoting revascularisation ( $\mathrm{Du}$ et al, 2008). HIF-1 $\alpha$ was moderately expressed in D2A1-wt, shMT1-mock and shMT1-40
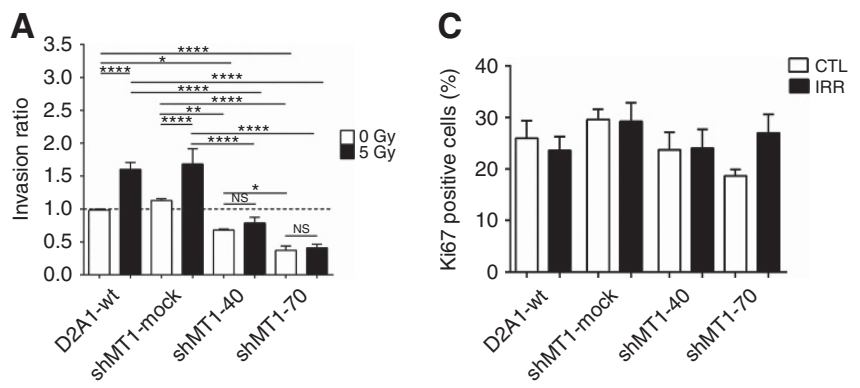

B

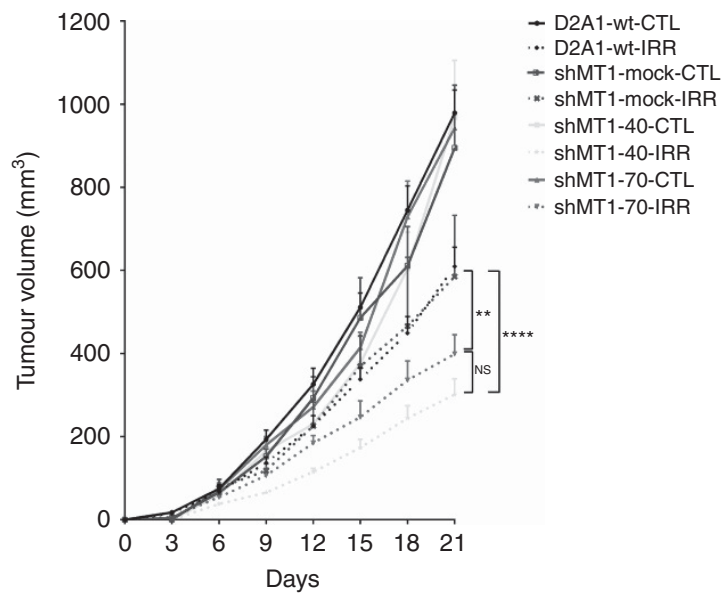

D

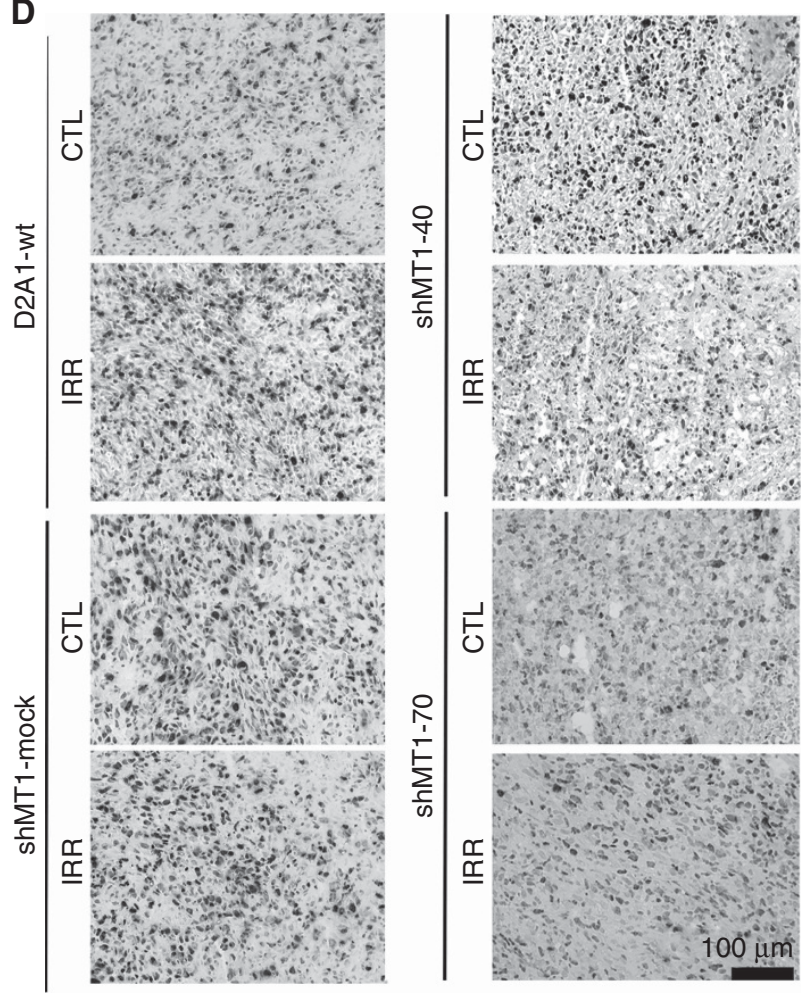

Figure 1. Effect of the pre-irradiated microenvironment on D2A1 breast cancer cell invasion and tumour growth assessed with cell lines downregulated for MT1-MMP. (A) Conditioned media from irradiated (5 Gy) 3 T3 fibroblasts was added in the lower compartment of the invasion chambers and used as chemoattractant for breast cancer cells added in the upper compartment. MT1-MMP downregulation completely blocked radiation enhancement of invasion of TNBC cell lines. The downregulation of the MT1-MMP also reduced the basal invasiveness of D2A1 cells. Decreases of $32 \%$ and $63 \%$ were observed for D2A1 shMT1-40 and D2A1 shMT1-70 cells $(P<0.0001 ; n=6)$. (B) D2A1 tumour volumes measured after implantation in pre-irradiated (dotted lines) or non-irradiated (solid lines) mammary glands of BALB/c mice. On day 21 , tumour volumes for all the cell lines were significantly reduced when implanted in pre-irradiated mammary glands, compared with non-irradiated mammary glands. Downregulation of MT1-MMP expression further reduced the tumour volume, but only in the pre-irradiated tissue. Volumes of shMT1-40 and shMT1-70 tumours were two-fold and 1.5-fold decreased compared with wild-type tumours ( $n=9$ to 21 tumours for each group). (C) Quantification of Ki67-positive cells by IHC on tumour frozen sections. No difference was observed ( $n=5$ to 11 tissue sections, each from a different tumour, were analysed for each condition). (D) Representative IHC images of Ki67 staining. CTL = control, IRR $=$ irradiated. ${ }^{\star} P<0.05,{ }^{\star *} P<0.01$ and $\star \star \star \star P<0.0001$. A full color version of this figure is available at the British Journal of Cancer journal online. 
A

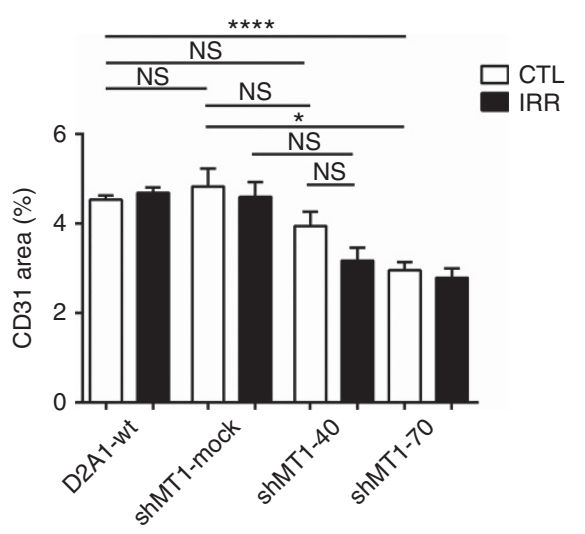

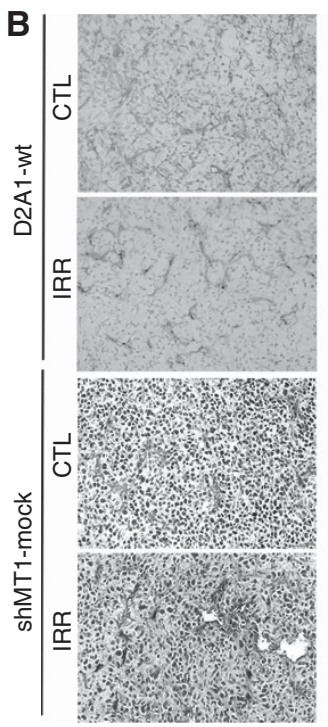

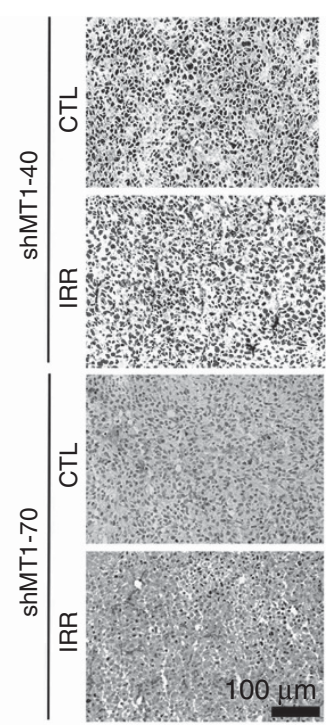

Figure 2. Vascularisation assessed in D2A1 tumour frozen sections. (A) Quantification of the blood vessel marker CD31 plotted as a percentage of stained area. Mammary gland irradiation did not significantly modify the CD31 staining in tumours, except for the shMT1-70 tumours in which a significant reduction was measured ( $n=3$ to 14 sections, each from a different tumour, was analysed for each condition). (B) Representative IHC images of $\mathrm{CD} 31$ staining. $C T L=$ control, IRR $=$ irradiated. ${ }^{\star} P<0.05$ and ${ }^{\star \star \star \star \star} P<0.0001$. A full color version of this figure is available at the British Journal of Cancer journal online.

tumour. As expected, a significant decrease of HIF- $1 \alpha$ expression was observed in shMT1-70 tumours, confirming the efficiency of the knockdown (Figure 3A and B). Interestingly, no difference in HIF- $1 \alpha$ expression was observed between tumour implanted in non-irradiated mammary glands with tumours implanted in the pre-irradiated microenvironment, even though control tumours were markedly bigger. These results suggest that the decrease of tumour volumes resulting from MT1-MMP knockdown when D2A1 cells are implanted in the pre-irradiated microenvironment is not related to angiogenesis neither than HIF- $1 \alpha$ expression.

Pre-irradiation of mammary gland modifies the MT1-MMP localisation in tumour cells. The diminution of MT1-MMP expression did not affect the morphology of the D2A1 cells, neither implanted in control nor in pre-irradiated mammary glands (Figure 3C). MT1-MMP was mainly located in the nucleus (black arrowheads) and cytoplasm but perinuclear staining was also observed in some cells (arrows). Interestingly, pre-irradiation of the mammary gland has modified MT1-MMP localisation in downregulated tumours, as shown by a lower accumulation in the nucleus (Figure $3 \mathrm{C}$ and $\mathrm{D}$ ). The number of negative nuclei was increased by 2.9 times $(P<0.0001)$ for both shMT1-40 and shMT1-70 tumours implanted in pre-irradiated mammary glands compared with non-irradiated mammary glands. This reduction of negative nuclei was not observed with the D2A1-wt and mock tumours implanted in the pre-irradiated mammary glands.

The possible endogenous expression of the MT1-MMP in the mammary gland as well as the possible increase of the protein stimulated by the pre-irradiated microenvironment were excluded since endogenous expression of the MT1-MMP in the normal mammary epithelium was not affected by radiation (Supplementary Figure $4 \mathrm{~A}-\mathrm{C}$ ). In the same way, irradiated microenvironments did not affect the expression of MT1-MMP in D2A1 cells implanted in the irradiated mammary glands (Supplementary Figure 4D). For these IHC assays, normal mouse mammary tissue was used as a positive control for the MT1-MMP antibody specificity (Supplementary Figure 5).

Reduction of MT1-MMP expression prevents radiationenhancement of CTC and lung metastases. Areas of mammary glands infiltrated by the D2A1 cells were measured in paraffin- embedded sections after H\&E staining. In the D2A1-wt and mock tumours implanted in non-irradiated mammary glands, no significant migration into the surrounding tissue was observed (Figure 4D, upper panel), while their migration was significantly increased when implanted in pre-irradiated mammary glands (D2A1-wt, $P=0.0220$; D2A1 shMT1-mock, $P=0.0433$; Figure $4 \mathrm{D}$, bottom panel). This adverse effect of radiation was completely blocked when the MT1-MMP expression was reduced in D2A1 cells (Figure 4E).

To determine the role of MT1-MMP in cell dissemination, CTC were quantified on days 4 and 7 after the implantation of the D2A1 MT1-MMP knockdown cell lines in the mammary fat pads (Figure 4A). Irradiation promoted the migration of D2A1 cells from the mammary gland to the blood vessels as shown by the higher number of CTC when the D2A1-wt and shMT1-mock cells were implanted. This increase was statistically significant on day 7 (2.5 times; $P<0.0001,2.4$ times; $P=0.009$ ). Conversely, the number of CTC was not increased when the MT1-MMP-deficient cells were implanted in irradiated mice compared with shamirradiated mice.

The increase of CTC induced by radiation was correlated with a higher number of lung metastases when mice were implanted with D2A1-wt or shMT1-mock cells $(P=0.0002 ; P=0.0048$; Figure 4B and $C)$. As expected, this stimulation by irradiating the mammary gland was not observed when the MT1-MMP-deficient cells were implanted. It is noteworthy that when implanted in non-irradiated mammary gland, the reduction of MT1-MMP expression did not significantly decrease the number of lung metastases as no difference was found for all sham group.

Effect of MT1-MMP on cell cycle distribution. Cancer cells can either migrate or proliferate, a phenomenon known as the migration/proliferation dichotomy (Giese et al, 1996). In our model, the FUCCI colourimetric vectors (Sakaue-Sawano et al, 2008) expressed by the D2A1 cells generate a green fluorescence when cells are in the $S / G_{2} / M$ phases (dividing cells) and red fluorescence for the $\mathrm{G}_{1} / \mathrm{G}_{\mathrm{O}}$ phases (non-dividing enriched cells). As MT1-MMP downregulation prevented radiation enhancement of CTC and lung metastases, we determined whether this stimulation was associated with enrichment to the $G_{1} / G_{O}$ phases, which would favour cancer cell migration. Pre-irradiation of mammary gland 
A

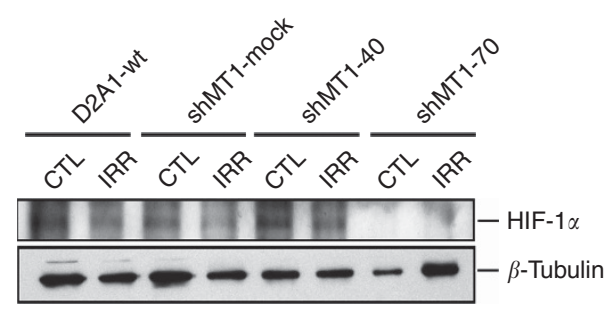

C

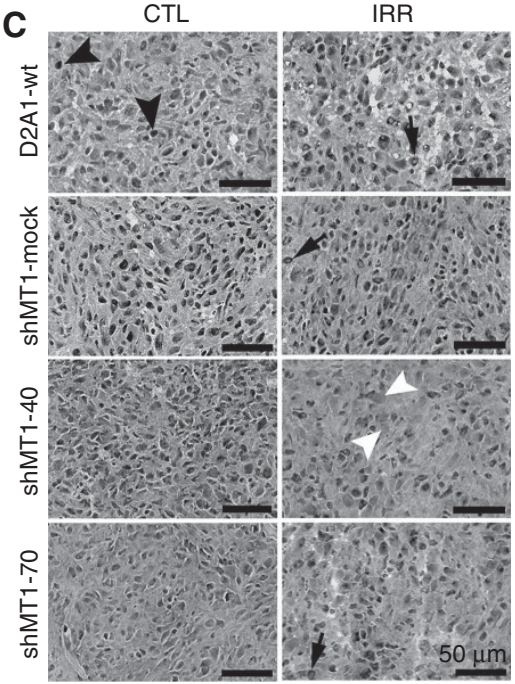

D

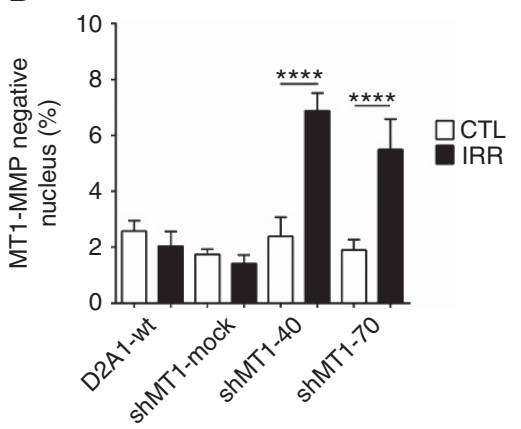

Figure 3. Hypoxia quantification assessed by HIF-1 $\alpha$ western blot and MT1-MMP localisation on tumour sections. (A) Representative western blot showing moderate HIF-1 $\alpha$ protein expression in D2A1-wt, shMT1-mock and shMT1-40 as well as a complete inhibition in shMT1-70 tumours. (B) Densitometry of HIF-1 $\alpha$ western blots from three independent experiments $(n=3$, each protein lysate is from a different tumour, for each condition). (C) Representative IHC images of MT1-MMP staining in frozen tumour sections. ( $n=3$ tissue sections, each from a different tumour, for each condition). (D) Quantification of the MT1-MMP negative nucleus. Black arrowheads=MT1-MMP positive nuclei, white arrowheads $=$ MT1-MMP negative nuclei, arrows = perinuclear staining. CTL $=$ control, IRR $=$ irradiated. ${ }^{\star \star} P<0.01,{ }^{\star \star \star} P<0.001$ and ${ }^{* \star \star \star} P<0.0001$. A full color version of this figure is available at the British Journal of Cancer journal online

increased D2A1 cells in the $\mathrm{G}_{1} / \mathrm{G}_{\mathrm{O}}$ phases by about $20 \%$ $(P<0.0001)$ for D2A1-wt and shMT1-mock cells (Figure 5). This increase of cells in $G_{1} / G_{O}$ phases (red cells) was associated with a decrease of dividing cells (green cells). Promotion in the $G_{1} / G_{O}$ phases induced by irradiated mammary gland was not observed in D2A1 cells downregulated for MT1-MMP. These results support that the pre-irradiated microenvironment promoted cell migration on a MT1-MMP-dependent basis.

\section{DISCUSSION}

The concept of radiation-induced invasion of cancer cells is generally accepted now (Jia et al, 2011; Chou et al, 2012; Desmarais et al, 2012; Bouchard et al, 2013; Moncharmont et al, 2014; Vilalta et al, 2014), but the underlying mechanism still need to be further investigated. For this purpose, the present study highlights the contribution of MT1-MMP in the promotion of TNBC invasion and formation of lung metastases by the irradiated stroma. As the D2A1 cells were not irradiated, our mouse model excludes the common hypothesis that this undesired effect was caused by new mutations in cancer cells induced by radiation. Indeed, our results showed that irradiation of the microenvironment is sufficient to trigger the progression of breast cancer, a phenomenon that was linked to the expression of MT1-MMP by the cancer cells. Main findings of our study are summarised in Figure 6.
Using in vitro assays and animal models, the ability of radiation to increase the invasiveness of human and mouse cancer cell lines was previously demonstrated (Park et al, 2006; Jia et al, 2011; Paquette et al, 2011, 2013; Chou et al, 2012; Bouchard et al, 2016). A limitation of the present study is that only one cell line was assessed. However, for in vivo experiments, syngeneic mouse models, as used in our study, should be used since radiationinduced lung metastasis development is strongly associated to the inflammatory response. For this reason, human xenografts implanted in immunodeficient mice would not be relevant. On the other hand, there is only one other mouse TNBC cell line commercially available, the $4 \mathrm{~T} 1$. The ability of radiation to stimulate their invasion was previously reported using invasion chambers (Bouchard et al, 2016).

The role of MT1-MMP in cancer invasion has already been reported in review articles (Seiki, 2003; Poincloux et al, 2009; Sato \& Takino, 2010). In tumour specimens from TNBC patients, elevated MT1-MMP expression was correlated with blood vessel invasion of cancer cells, poor prognosis and high incidence of distant metastasis relative to other breast cancer subtypes (Perentes et al, 2011). Supporting the role of MT1-MMP, its downregulation reduced the invasiveness of the human TNBC MDA-MB-231 cells in vitro (Jiang et al, 2006), and decreased the number of lung metastases in mice following tumour implantation (Perentes et al, 2011). However, the role of MT1-MMP has not been investigated in the presence of radiation yet, and we have designed this study to 
A

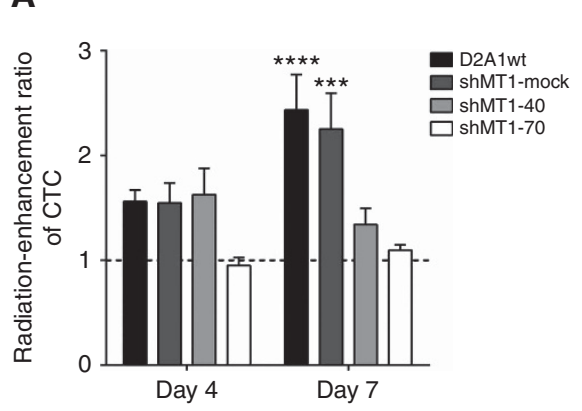

C
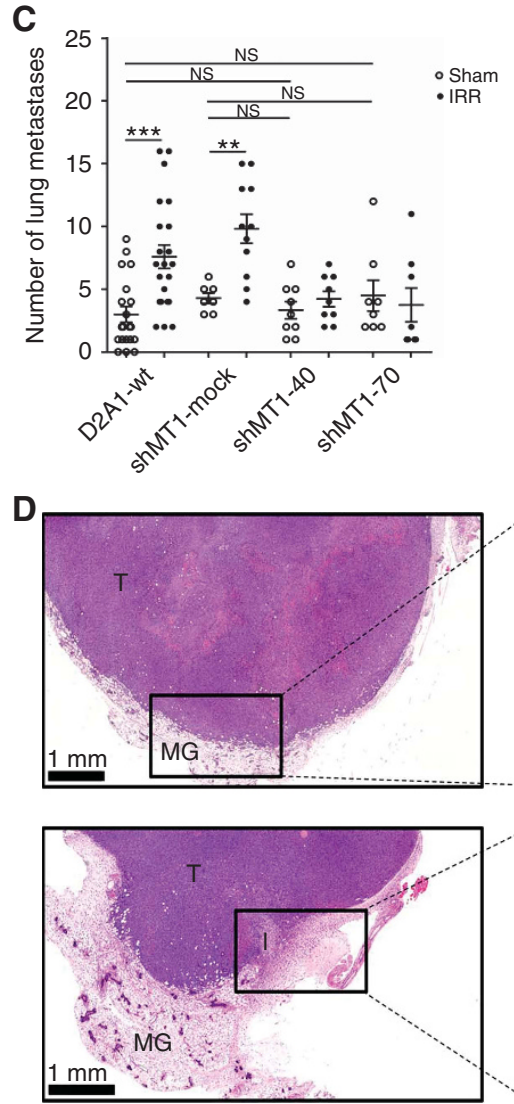

B
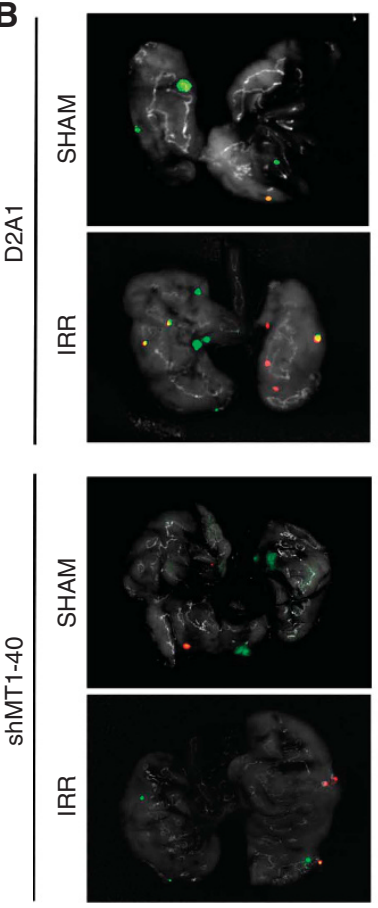
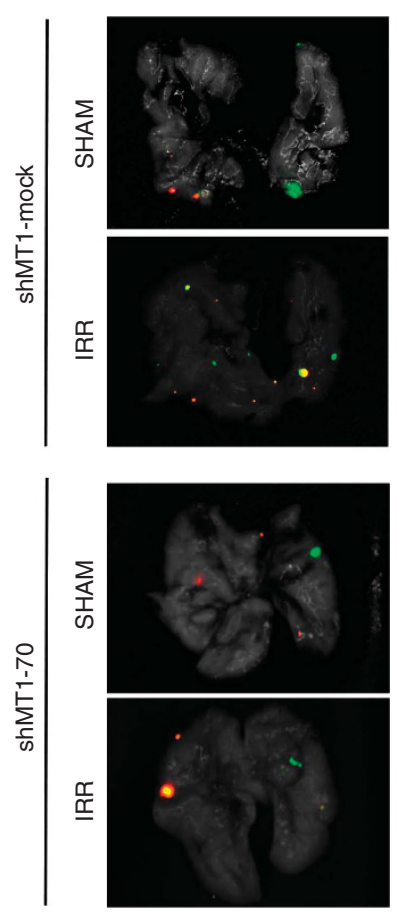

E
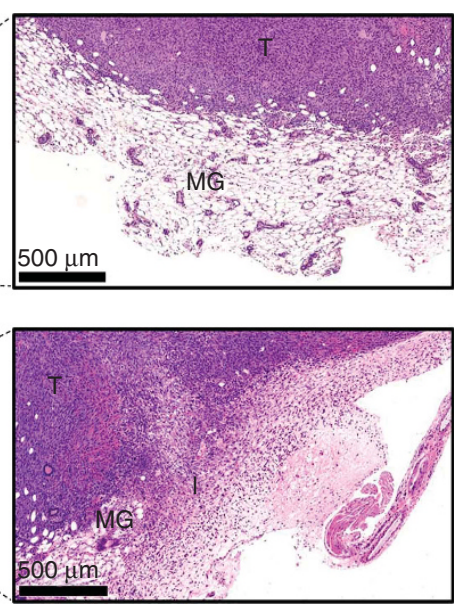

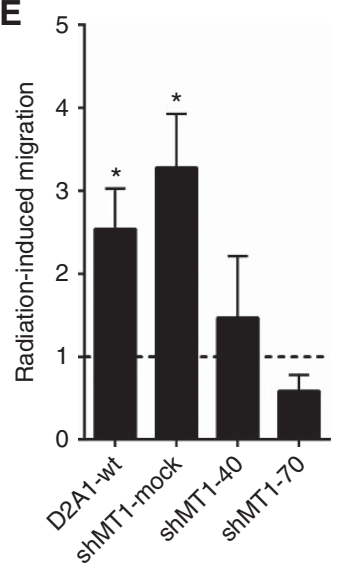

Figure 4. Downregulation of MT1-MMP expression has inhibited radiation enhancement of cancer cell invasion, CTC and lung metastases. (A) Quantification of CTC in blood samples of sham and irradiated mice. Results were plotted as radiation enhancement of CTC number and calculated as the mean number of CTC in the blood of irradiated animals divided by the mean number of CTC quantified in the blood of sham animals. The increase of CTC was statistically significant only on day 7 for D2A1-wt and shMT1-mock cells. This radiation enhancement of the CTC was not observed in the shMT1-40 and shMT1-70 cells implanted in mice; $n=3$ to 11 mice for each condition. (B) Optical imaging of lung metastases on the day of killing, 21 days after tumour implantation. (C) Quantification of the number of lung metastases. The number of lung metastases was increased in irradiated mice compared with sham groups for D2A1-wt and shMT1-mock cells. This radiation enhancement of lung metastases was not observed with the shMT1-40 and shMT1-70 cells ( $n=8$ to 23 mice for each condition). Sham mice: non-irradiated animals with tumour implantation in the right and left third mammary glands. Irradiated animals: pre-irradiation of the third right mammary gland followed by tumour implantation in the right and left third mammary glands. (D) H\&E staining of D2A1 tumour sections showing that radiation-induced migration in mammary glands was MT1-MMP-dependent. (E) Quantification of D2A1 tumour migration. Migration ratios were calculated as infiltration area divided by the primary tumour area. Migration ratios were higher in tumours implanted in pre-irradiated mammary glands for the D2A1-wt and shMT1-mock tumours. This radiation enhancement of migration was not observed in the shMT1-40 and shMT1-70 tumours ( $n=2$ to 6 tissue sections, each from a different tumour, for each condition). I= infiltration; $M G=$ mammary gland; $T=D 2 A 1$ tumour. ${ }^{\star} P<0.05,{ }^{\star} P<<0.01$, $\star \star \star P<0.001$ and ${ }^{* \star \star \star} P<0.0001$.

determine whether radiation can promote cancer progression in a TNBC animal model.

Here, using a TNBC mouse model, pre-irradiation of the mammary gland, followed by the implantation of D2A1 cancer cells has increased the number of migrating cells in the irradiated microenvironment at the expense of tumour growth. This can be associated with the migration/proliferation dichotomy, which was previously described as mutually exclusive or as a «Go or Grow» phenomenon (Giese et al, 1996; Bouchard et al, 2013, 2016). Consequently, this enrichment of migrating cells induced by radiation could explain the reduction of tumour growth observed when the D2A1-wt and shMT1-mock cell lines were implanted in the pre-irradiated mammary glands. However, reduction of tumour growth was more important in the MT1-MMP 

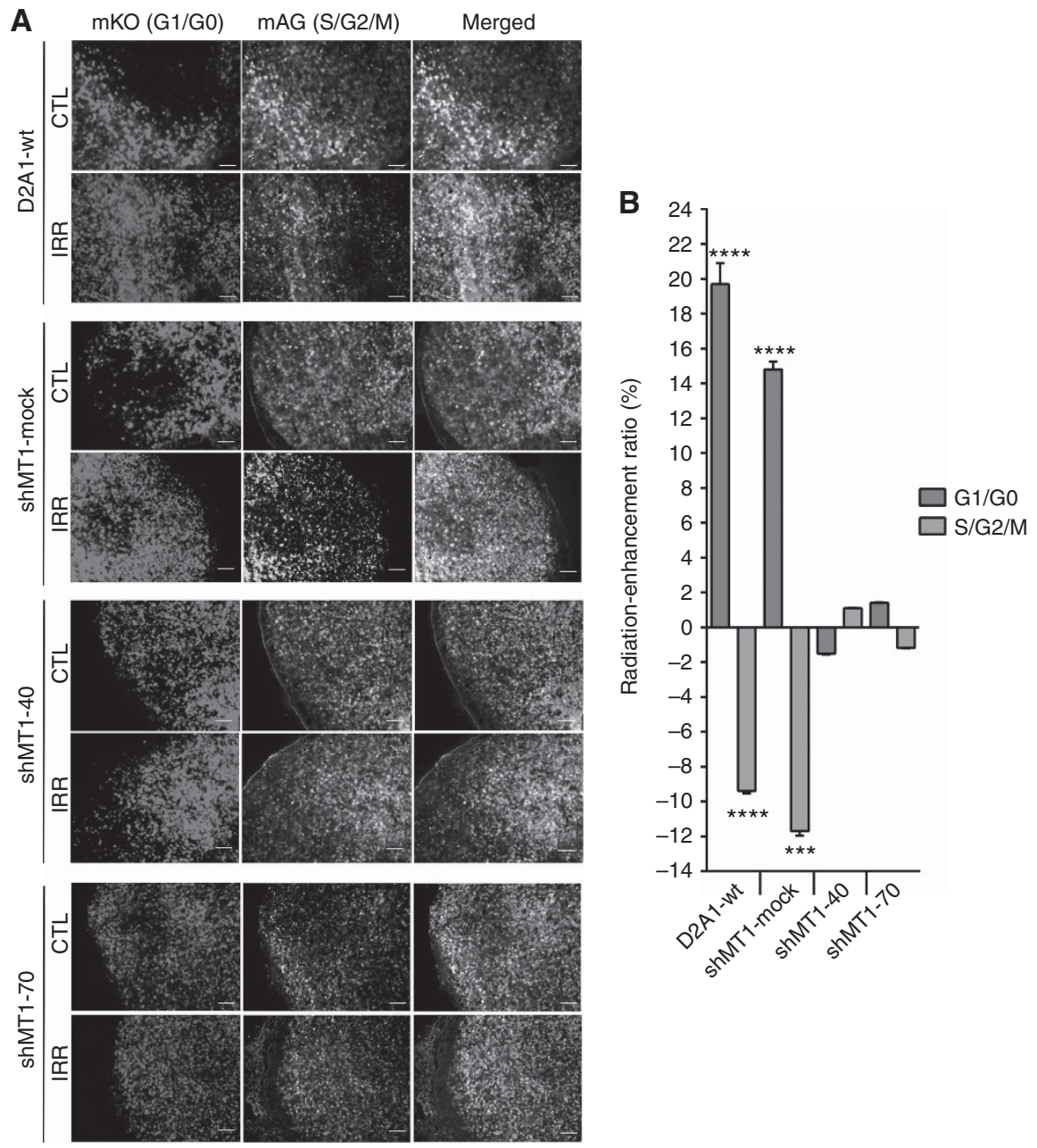

Figure 5. Effect of MT1-MMP downregulation on the cell cycle distribution in D2A1 FUCCI tumours. (A) Representative fluorescence images of frozen mammary tumour sections that were used to quantify cancer cells in S/G2/M (green) or G1/G0 (red) phases (magnification $\times 42$, scale bar $=200 \mu \mathrm{m}$ ). (B) Effect of radiation on the cell cycle distribution plotted as a radiation-enhancement ratio of red and green cells in percentages. A significant radiation-enhancement of G1/G0 (red) cells was observed for D2A1-wt and shMT1-mock tumours. MT1-MMP downregulation completely prevented this augmentation ( $n=6$ to 13 for each condition). ${ }^{\star \star \star} P<0.001$ and ${ }^{\star \star \star \star} P<0.0001$. A full color version of this figure is available at the British Journal of Cancer journal online.

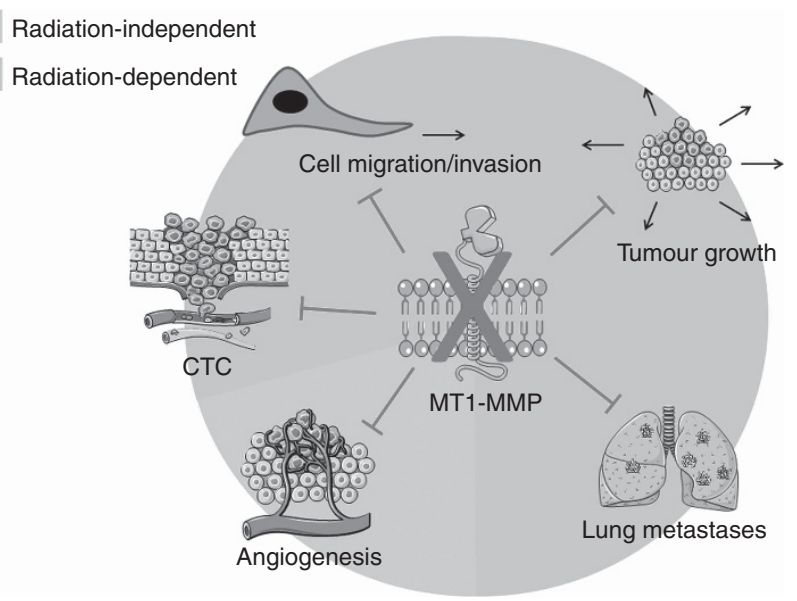

Figure 6. Summary of the proposed involvement of MT1-MMP in radiation-stimulated development of lung metastases. A full color version of this figure is available at the British Journal of Cancer journal online

downregulated tumours, although radiation-enrichment of migrating D2A1 cells in the surrounding tissue was inhibited. On the other hand, downregulation of MT1-MMP did not affect tumour volume when the D2A1 sublines knockdown for MT1-MMP were implanted in non-irradiated mammary glands. Similar results were reported by other groups when comparing MDA-MB-231 cells wild-type and knockdown for MT1-MMP in non-irradiated conditions (Jiang et al, 2006; Perentes et al, 2011). Reduction of tumour growth in the pre-irrradiated mammary glands can thus not be solely associated with a downregulation of the MT1-MMP as the pre-irradiated microenvironment was essential to slow down tumour growth.

As MT1-MMP expression can promote angiogenesis (Sounni et al, 2002), we have determined whether a decrease of angiogenesis as well as hypoxia could have explained the reduction of tumour growth in MT1-MMP knockdown tumours implanted in irradiated mammary glands. No significant reduction of the CD31 (endothelial cell marker) area was measured between tumours either implanted in irradiated or non-irradiated mammary glands. Similar results were observed for HIF- $1 \alpha$ measured expression in tumour lysates. Although a significant diminution of hypoxia was observed in shMT1-70 tumours, no difference was observed between control tumours and those implanted in irradiated mammary glands. These results exclude that alterations in angiogenesis and hypoxia were associated with radiationinduced invasion of D2A1 cells in our model. In what way the irradiated microenvironment significantly reduce tumour growth 
without favouring the infiltration of D2A1 sublines knockdown for MT1-MMP remains to be determined.

Our study has also contributed to further understand the effect of radiation on blood vessels and its impact on the increase of CTC related to MT1-MMP expression. Pre-irradiation of the mammary gland increased the number of CTC after D2A1-wt and mock cell implantation into the mammary fat pads. This stimulation cannot be solely associated with significant radiation-induced blood vessel damages as downregulation of MT1-MMP completely inhibited the radiation-increased number of CTC.

The increase of CTC and lung metastases observed with high MT1-MMP expressing cell lines (wild type and mock) could have been related to a stimulation of MMP-2 activity (the main substrate of MT1-MMP also known for its role in cancer invasion). However, we have shown in a previous study (Bouchard et al, 2013) using the same mouse model that no increase of MMP-2 was measured in the irradiated mammary glands 6 or $24 \mathrm{~h}$ after the last fraction of irradiation $(4 \times 6 \mathrm{~Gy} ; 24 \mathrm{~h}$ intervals), and neither in D2A1 tumours implanted in pre-irradiated mammary glands. Therefore, these results suggest an alternative role of MT1-MMP may occur in the phenomenon of radiation-induced invasion stimulated by the microenvironment. On the other hand, irradiation of a D2A1 tumour implanted in the mammary gland (unpublished results) was associated with an increase of MMP-2 activity. These results suggest that the increased activity of the MMP-2 from the irradiated cancer cells rather than the irradiated microenvironment could contribute to TNBC progression.

Alternatively, the role of MT1-MMP in cancer cell invasion could be related to the regulation of genes associated with the epithelial-mesenchymal transition (EMT), as the induction of EMT in irradiated human colorectal cancer has been associated with an enhancement of their invasiveness (Yan et al, 2015; Diao et al, 2016). However, the role of MT1-MMP in EMT induction related to the invasion of TNBC cells remains to be determined.

In our study, MT1-MMP mRNA, protein expression and enzymatic activity (by measuring the activation of proMMP-2 to MMP-2) were quantified. MT1-MMP mRNA was downregulated by $40 \%$ and $70 \%$, respectively in the D2A1 shMT1-40 and shMT170 cell homogenates but the protein expression reached $18 \%$ and $40 \%$ reduction when quantified in tumour homogenates. On the other hand, a 50\% reduction of the enzymatic activity of MT1-MMP was measured in their tumour homogenates. These discrepancies highlight that MT1-MMP expression and activity depend on the tumour microenvironment, and its accurate quantification can be complex. Our results also support the idea that only a partial downregulation of the MT1-MMP is required to completely inhibit the development of lung metastases induced by the pre-irradiated mammary gland. This result suggests the existence of a threshold of MT1-MMP expression above which the risk of early recurrence increases but this effect is not linearly correlated with its expression. Consequently, it may be difficult to predict which TNBC patients are at high risk of recurrence based solely on the expression of MT1-MMP in their tumour specimens.

Our results suggest that the predictive value of MT1-MMP may also be depending on its localisation in tumour cells. MT1-MMP is located at the membrane and cytoplasm of normal and tumour cells (Ip et al, 2007). In hepatocellular carcinoma, overexpression of MT1-MMP and its atypical localisation in the nucleus were correlated with promotion of metastasis and poor overall survival (Ip et al, 2007). Although the general mechanism is still partially understood, MT1-MMP appears to be associated with chromosomal instability (Golubkov et al, 2005), which supports its role in the nucleus to increase cancer aggressiveness. In our mouse model, localisation of MT1-MMP in the nucleus was also observed for D2A1 tumours suggesting that it may also have a predictive value for metastasis development in TNBC. Interestingly, the knockdown of MT1-MMP has reduced the accumulation of MT1-MMP in the nucleus, but only when the D2A1 cells were implanted in the preirradiated microenvironment. Moreover, this reduction of nuclear MT1-MMP was associated with the inhibition of radiation-stimulated metastasis development. However, further studies are needed regarding how the irradiated microenvironment prevents this translocation only in the presence of a low expression of MT1-MMP.

In conclusion, this study highlights that MT1-MMP is associated with metastasis formation when tumours are implanted in preirradiated mammary gland. The next step will be to validate this effect of radiation when TNBC tumours expressing a lower level of MT1MMP are directly irradiated. These results will then support to determine whether the expression and cellular localisation of MT1MMP in TNBC biopsies could be used as a tool to predict which TNBC patients are at high risk of early recurrence.

\section{ACKNOWLEDGEMENTS}

BP, RB and CS are members of the Fonds de la Recherche en Santé du Québec (FRSQ)-funded Centre de recherche CHUS. GB held a scholarship from FRSQ (grant \# 27479). The authors thank the medical physicists Patrick Delage and Vincent-Hubert Tremblay for their very helpful dosimetry calculations for mice irradiation. The authors also thank the Electron Microscopy and Histology Research Core of the FMSS at the Universite de Sherbrooke for their histology, electron microscopy and phenotyping services as well as the Plateforme d'Analyse et de Visualisation d'Images (PAVI) for the automated quantification of the results. This research project was supported by the Canadian Institutes of Health Research (grant \#184671).

\section{CONFLICT OF INTEREST}

The authors declare no conflict of interest.

\section{AUTHOR CONTRIBUTIONS}

GB performed all animal experiments, analyses, result interpretations and drafted the manuscript. HT generated FUCCI cells and performed in vitro experiments. GB, CS, RB and BP conceptualised the study. SG contributed to pathological analysis. BP, CS, RB and SG contributed to writing and revising the manuscript. All the authors contributed to critical analysis and approval of the final manuscript.

\section{REFERENCES}

Ager EI, Kozin SV, Kirkpatrick ND, Seano G, Kodack DP, Askoxylakis V, Huang Y, Goel S, Snuderl M, Muzikansky A, Finkelstein DM, Dransfield DT, Devy L, Boucher Y, Fukumura D, Jain RK (2015) Blockade of MMP14 activity in murine breast carcinomas: implications for macrophages, vessels, and radiotherapy. J Natl Cancer Inst 107(4): djv017.

Bouchard G, Bouvette G, Therriault H, Bujold R, Saucier C, Paquette B (2013) Pre-irradiation of mouse mammary gland stimulates cancer cell migration and development of lung metastases. Br J Cancer 109: 1829-1838.

Bouchard G, Therriault H, Geha S, Berube-Lauziere Y, Bujold R, Saucier C, Paquette B (2016) Stimulation of triple negative breast cancer cell migration and metastases formation is prevented by chloroquine in a pre-irradiated mouse model. BMC Cancer 16: 361.

Chou CH, Teng C-M, Tzen K-Y, Chang Y-C, Chen J-H, Cheng JC-H (2012) MMP-9 from sublethally irradiated tumor promotes Lewis lung carcinoma cell invasiveness and pulmonary metastasis. Oncogene 31: 458-468.

Desmarais G, Charest G, Fortin D, Bujold R, Mathieu D, Paquette B (2015) Cyclooxygenase-2 inhibitor prevents radiation-enhanced infiltration of F98 glioma cells in brain of Fischer rat. Int J Radiat Biol 91: 624-633. 
Desmarais G, Fortin D, Bujold R, Wagner R, Mathieu D, Paquette B (2012) Infiltration of glioma cells in brain parenchyma stimulated by radiation in the F98/Fischer rat model. Int J Radiat Biol 88: 565-574.

Diao WL, Luo L, Luo Q (2016) Effect of X-ray irradiation on epithelialmesenchymal transition of colorectal cancer SW480 cells. J Biol Regul Homeost Agents 30: 553-558.

Du R, Lu KV, Petritsch C, Liu P, Ganss R, Passegué E, Song H, VandenBerg S, Johnson RS, Werb Z, Bergers G (2008) HIFl $\alpha$ induces the recruitment of bone marrow-derived vascular modulatory cells to regulate tumor angiogenesis and invasion. Cancer Cell 13: 206-220.

Egeblad M, Werb Z (2002) New functions for the matrix metalloproteinases in cancer progression. Nat Rev Cancer 2: 161-174.

Giese A, Loo MA, Tran N, Haskett D, Coons SW, Berens ME (1996) Dichotomy of astrocytoma migration and proliferation. Int J cancer 67: 275-282.

Golubkov VS, Boyd S, Savinov AY, Chekanov AV, Osterman AL, Remacle A, Rozanov DV, Doxsey SJ, Strongin AY (2005) Membrane type-1 matrix metalloproteinase (MT1-MMP) exhibits an important intracellular cleavage function and causes chromosome instability. J Biol Chem 280: 25079-25086.

Hotary KB, Yana I, Sabeh F, Li XY, Holmbeck K, Birkedal-Hansen H, Allen ED, Hiraoka N, Weiss SJ (2002) Matrix metalloproteinases (MMPs) regulate fibrin-invasive activity via MT1-MMP-dependent and -independent processes. J Exp Med 195: 295-308.

Ip YC, Cheung ST, Fan ST (2007) Atypical localisation of membrane type 1-matrix metalloproteinase in the nucleus is associated with aggressive features of hepatocellular carcinoma. Mol Carcinog 46: 225-230.

Itoh Y (2006) MT1-MMP: a key regulator of cell migration in tissue. IUBMB Life 58: 589-596.

Jia J-B, Wang W-Q, Sun H-C, Liu L, Zhu X-D, Kong L-Q, Chai Z-T, Zhang W, Zhang J-B, Xu H-X, Zeng Z-C, Wu W-Z, Wang L, Tang Z-Y (2011) A novel tripeptide, tyroserleutide, inhibits irradiation-induced invasiveness and metastasis of hepatocellular carcinoma in nude mice. Invest New Drugs 29: 861-872.

Jiang WG, Davies G, Martin TA, Parr C, Watkins G, Mason MD, Mansel RE (2006) Expression of membrane type-1 matrix metalloproteinase, MT1-MMP in human breast cancer and its impact on invasiveness of breast cancer cells. Int J Mol Med 17: 583-590.

Langlands FE, Horgan K, Dodwell DD, Smith L (2013) Breast cancer subtypes: response to radiotherapy and potential radiosensitisation. Br J Radiol 86: 20120601.

Lemay R, Archambault M, Tremblay L, Bujold R, Lepage M, Paquette B (2011) Irradiation of normal mouse tissue increases the invasiveness of mammary cancer cells. Int J Radiat Biol 87: 472-482.

Li Y, Cai G, Yuan S, Jun Y, Li N, Wang L, Chen F, Ling R, Yun J (2015) The overexpression membrane type 1 matrix metalloproteinase is associated with the progression and prognosis in breast cancer. Am J Transl Res 7: 120-127.

Loibl S, Jackisch C, Lederer B, Untch M, Paepke S, Kummel S, Schneeweiss A, Huober J, Hilfrich J, Hanusch C, Gerber B, Eidtmann H, Denkert C, Costa SD, Blohmer JU, Nekljudova V, Mehta K, von Minckwitz G (2015) Outcome after neoadjuvant chemotherapy in young breast cancer patients: a pooled analysis of individual patient data from eight prospectively randomized controlled trials. Breast Cancer Res Treat 152: 377-387.

Moncharmont C, Levy A, Guy J-B, Falk AT, Guilbert M, Trone J-C, Alphonse G, Gilormini M, Ardail D, Toillon R-A, Rodriguez-Lafrasse C, Magné N (2014) Radiation-enhanced cell migration/invasion process: a review. Crit Rev Oncol Hematol 92: 133-142.

Paquette B, Therriault H, Desmarais G, Wagner R, Royer R, Bujold R (2011) Radiation-enhancement of MDA-MB-231 breast cancer cell invasion prevented by a cyclooxygenase-2 inhibitor. Br J Cancer 105: 534-541.

Paquette B, Therriault H, Wagner JR (2013) Role of interleukin-1 $\beta$ in radiation-enhancement of MDA-MB-231 breast cancer cell invasion. Radiat Res 180: 292-298.

Park CM, Park MJ, Kwak HJ, Lee Hc, Kim MS, Lee SH, Park IC, Rhee CH, Hong SI (2006) Ionizing Radiation Enhances Matrix Metalloproteinase-2
Secretion and Invasion of Glioma Cells through Src/Epidermal Growth Factor Receptor-Mediated p38/Akt and Phosphatidylinositol 3-Kinase/Akt Signaling Pathways. Cancer Res 66: 8511-8519.

Perentes JY, Kirkpatrick ND, Nagano S, Smith EY, Shaver CM, Sgroi D, Garkavtsev I, Munn LL, Jain RK, Boucher Y (2011) Cancer cell-associated MT1-MMP promotes blood vessel invasion and distant metastasis in triple-negative mammary tumors. Cancer Res 71: 4527-4538.

Podo F, Buydens LM, Degani H, Hilhorst R, Klipp E, Gribbestad IS, Van Huffel S, van Laarhoven HW, Luts J, Monleon D, Postma GJ, Schneiderhan-Marra N, Santoro F, Wouters H, Russnes HG, Sorlie T, Tagliabue E, Borresen-Dale AL, Consortium F (2010) Triple-negative breast cancer: present challenges and new perspectives. Mol Oncol 4: 209-229.

Poincloux R, Lizárraga F, Chavrier P (2009) Matrix invasion by tumor cells: a focus on MT1-MMP trafficking to invadopodia. J Cell Sci 122: 3015-3024.

Qian LW, Mizumoto K, Urashima T, Nagai E, Maehara N, Sato N, Nakajima M, Tanaka M (2002) Radiation-induced increase in invasive potential of human pancreatic cancer cells and its blockade by a matrix metalloproteinase inhibitor, CGS27023. Clin Cancer Res 8: 1223-1227.

Rofstad EK, Mathiesen B, Galappathi K (2004) Increased metastatic dissemination in human melanoma xenografts after subcurative radiation treatment: radiation-induced increase in fraction of hypoxic cells and hypoxia-induced up-regulation of urokinase-type plasminogen activator receptor. Cancer Res 64: 13-18.

Sakaue-Sawano A, Kurokawa H, Morimura T, Hanyu A, Hama H, Osawa H, Kashiwagi S, Fukami K, Miyata T, Miyoshi H, Imamura T, Ogawa M, Masai H, Miyawaki A (2008) Visualizing spatiotemporal dynamics of multicellular cell-cycle progression. Cell 132: 487-498.

Sato H, Takino T (2010) Coordinate action of membrane-type matrix metalloproteinase-1 (MT1-MMP) and MMP-2 enhances pericellular proteolysis and invasion. Cancer Sci 101: 843-847.

Seiki M (2003) Membrane-type 1 matrix metalloproteinase: a key enzyme for tumor invasion. Cancer Lett 194: 1-11.

Sounni NE, Devy L, Hajitou A, Frankenne F, Munaut C, Gilles C, Deroanne C, Thompson EW, Foidart JM, Noel A (2002) MT1-MMP expression promotes tumor growth and angiogenesis through an up-regulation of vascular endothelial growth factor expression. Faseb j 16: 555-564.

Speake WJ, Dean RA, Kumar A, Morris TM, Scholefield JH, Watson SA (2005) Radiation induced MMP expression from rectal cancer is short lived but contributes to in vitro invasion. Eur J Surg Oncol 31: 869-874.

Vilalta M, Rafat M, Giaccia AJ, Graves EE (2014) Recruitment of circulating breast cancer cells is stimulated by radiotherapy. Cell Rep 8: 402-409.

Villarreal-Garza C, Bargallo-Rocha JE, Soto-Perez-de-Celis E, Lasa-Gonsebatt F, Arce-Salinas C, Lara-Medina F, Reynoso-Noveron N, Matus-Santos J, Cabrera P, Alvarado-Miranda A, Mohar A (2016) Real-world outcomes in young women with breast cancer treated with neoadjuvant chemotherapy. Breast Cancer Res Treat 157: 385-394.

Wang JL, Sun Y, Wu S (2000) Gamma-irradiation induces matrix metalloproteinase II expression in a p53-dependent manner. Mol Carcinog 27: 252-258.

Wild-bode C, Weller M, Rimner A, Dichgans J, Wick W (2001) Sublethal irradiation promotes migration and invasiveness of glioma Cells: implications for radiotherapy of human glioblastoma. Cancer Res 61: 2744-2750.

Yan T, Lin Z, Jiang J, Lu S, Chen M, Que H, He X, Que G, Mao J, Xiao J, Zheng Q (2015) MMP14 regulates cell migration and invasion through epithelial-mesenchymal transition in nasopharyngeal carcinoma. Am J Transl Res 7: 950-958.

This work is published under the standard license to publish agreement. After 12 months the work will become freely available and the license terms will switch to a Creative Commons AttributionNonCommercial-Share Alike 4.0 Unported License.

Supplementary Information accompanies this paper on British Journal of Cancer website (http://www.nature.com/bjc) 